\title{
Morphogenesis of Biologically Active Interfollicular Epidermis from Human Embryonic Stem Cell-derived Keratinocytes
}

\author{
Rasmussen CA, Schlosser SJ and Allen-Hoffmann BL*
}

School of Medicine \& Public Health, 5640 Medical Sciences Center, Madison, WI 53706, USA

\begin{abstract}
For patients suffering from extensive burns or chronic wounds, allogeneic cell-based therapies provide a means to restore viability and function to severely damaged cutaneous tissues. Pluripotent stem cells have been proposed as an allogeneic source for regenerative medicine applications, such as those required for burn and wound management. Ultimately the clinical utility of pluripotent stem cells relies on the ability to direct differentiation into the desired cell lineage, facilitate appropriate tissue formation, and confirm tissue-specific biological activity. We examined the capacity of human embryonic stem cell-derived keratinocytes (hES-DK) to undergo morphogenesis and form biologically active interfollicular epidermis using methods routinely employed to engineer skin substitutes for clinical applications. Throughout directed differentiation, the orderly sequence of epidermal gene expression mimicked the progression of fetal skin development. When introduced into three-dimensional organotypic culture, hES-DK cells formed a pluristratified tissue with architecture similar to that of the interfollicular epidermis. In hES-DK tissue the expression and localization of cell-cell adhesion proteins, markers of both early- and late-stage keratinocyte terminal differentiation, and host defense peptides were comparable to the patterns observed in keratinized stratified squamous epithelia generated from epidermal keratinocytes. Despite similar tissue morphology, functional analysis revealed that hES-DK tissues did not display robust cutaneous barrier function. However, hES-DK tissues were shown to possess antimicrobial activity, which represents the first demonstration of biological activity in a keratinized stratified squamous epithelium generated from a pluripotent stem cell-derived source. The successful generation of biologically active hES-derived stratified squamous epithelia represents a significant advance in the development of a hES-derived bioengineered human organ for clinical use.
\end{abstract}

Keywords: Regenerative medicine; Allogeneic cell-based therapy; Human Embryonic Stem cell-derived keratinocyte; hES-derived; Biologically active interfollicular epidermis

\section{Introduction}

The utility of pluripotent stem cells, such as human embryonic stem (hES) cells and human induced pluripotent stem (hiPS) cells, for regenerative medicine applications relies on the cells' ability to differentiate into clinically relevant cell populations and form constructs that recapitulate the structure and function of the intended organ system. Directed differentiation protocols have been employed to generate multiple cell types, including those of neural, hematopoietic, cardiac, and pancreatic lineages $[1,2]$, as well as epithelial lineages, including keratinocytes [3-9]. For patients suffering from extensive burns or chronic wounds, allogeneic cell-based therapies provide a means to restore the viability and/or function of affected tissues [10]. Fundamentally, stem cell therapy in burn and wound management requires a consistent source of keratinocytes with the capacity to fully restore functional epidermis. To date, a thorough characterization of the structural properties and, more importantly, the biological activities of keratinized stratified squamous epithelia generated from a pluripotent stem cell source has not been reported.

Initial studies describing the derivation of keratinocyte precursors from hES cells relied on spontaneous differentiation events such as teratoma or embryoid body formation [3-5]. The resulting heterogeneous populations displayed sporadic differentiation into a keratinocyte lineage as evidenced by expression of markers such as keratin 14 (K14) or yielded keratinocyte-like cells with limited expansion potential. The efficiency of selecting K14+ keratinocyte-like cells was subsequently enhanced by treating with retinoic acid (RA) and bone morphogenic protein-4 (BMP4) [6]. Serial cultivation yielded a relatively pure population of keratinocyte-like cells which expressed several proteins associated with early-stage keratinocyte terminal differentiation in monolayer culture. In 2011, a differentiation protocol employing RA and BMP4 was also reported to facilitate the generation of K14-expressing keratinocytes from hiPS cells [7]. Differentiation methods using RA and Activin [8], as well as ascorbic acid and BMP4 [9], have also been shown to generate K14+ keratinocytes from hES cells provided the specified cultivation steps were adhered to.

Organotypic culturing techniques, which promote full stratification and differentiation of epidermal and oral keratinocytes under in vivolike conditions, have fostered the development of the first engineered human tissues for clinical use [11-13]. These specialized techniques have enabled the formation of three-dimensional epithelial tissue constructs from hES-derived cell populations [9,14-16]. Dabelstein et al. [14] first demonstrated epithelial tissue morphogenesis using hESderived, keratinocyte-like cells transduced with the oncogenic E6 and E7 genes of HPV16 to enable serial cultivation [14]. Three-dimensional tissue has also been generated from hiPS-derived keratinocytes and, although characterization was limited, was shown to express the

*Corresponding author: B. Lynn Allen-Hoffmann, Department of Pathology and Laboratory Medicine, 5605 Medical Sciences Center, 1300 University Avenue, Madison, WI 53706, USA, Tel: 608 262-2884; Fax: 608 265-3301; E-mail: blallenh@wisc.edu

Received November 09, 2013; Accepted January 24, 2014; Published January 27,2014

Citation: Rasmussen CA, Schlosser SJ, Allen-Hoffmann BL (2014) Morphogenesis of Biologically Active Interfollicular Epidermis from Human Embryonic Stem Cell-derived Keratinocytes. J Stem Cell Res Ther 4: 163. doi:10.4172/21577633.1000163

Copyright: (C) 2014 Rasmussen CA, et al. This is an open-access article distributed under the terms of the Creative Commons Attribution License, which permits unrestricted use, distribution, and reproduction in any medium, provided the original author and source are credited. 
terminal differentiation markers keratin 1 (K1) and loricrin [7]. Metallo et al. [6] utilized hES-derived cells to generate a tissue that, despite several atypical characteristics, displayed many morphological hallmarks of keratinized stratified squamous epithelia [15]. Recently, Leydon and coworkers demonstrated production of a non-keratinized stratified squamous epithelium from hES-derived simple epithelial cells created through employing the initial stages of Metallo's differentiation method [16]. This tissue was shown to differentially express a subset of proteins in a pattern consistent with vocal fold epithelium. In 2009, Guenou et al. [9] formed three-dimensional tissue from a hES-derived cell source that displayed both the characteristic morphology of keratinized stratified squamous epithelia and appropriate localization of several keratinocyte terminal differentiation markers [9]. A thorough characterization of the distinctive structural and functional proteins typical of a keratinized stratified squamous epithelium generated from both a hES or hiPS cell-derived source and the associated biological activities, has not yet been reported.

Here we describe the morphogenesis of biologically active keratinized stratified squamous epithelia from hES-derived keratinocytes (hES-DK). Treatment of hES cells with RA alone did not induce expression of K14 however, serial cultivation on gelatincoated plates selected for cells expressing key keratinocyte markers. This hES-DK population was capable of forming a pluristratified epithelium when cultivated under organotypic conditions. Epidermal morphogenesis was confirmed by comprehensively evaluating the expression and appropriate localization of proteins associated with the structure and function of keratinized stratified squamous epithelia, including cell-cell adhesion proteins, markers of both early- and latestage keratinocyte terminal differentiation, and host defense peptides (HDPs). The epidermis serves as a barrier to transepithelial water loss and functions to prevent bacterial invasion through secretion of HDPs. Although hES-DK tissue did not display cutaneous barrier function, we found that hES-derived epidermal tissue possessed antimicrobial activity, which has not previously been reported in a hES cell-derived tissue. These findings suggest that a well-defined hES-derived cell population, as confirmed by the expression of key epidermal markers, is required for the morphogenesis of biologically active interfollicular epidermis.

\section{Materials and Methods}

\section{Monolayer and organotypic cell culture methods}

hES cells (H9) [17] were obtained from the WiCell Research Institute (Madison, WI). Undifferentiated hES cells were cultivated on mitomycin C-inactivated murine fibroblasts in hES growth medium [4]. For passaging, cells of the feeder layer were dislodged by pipetting and rinsed free, facilitating hES cell harvest by manual colony dissociation. For directed differentiation, feeder cells were removed and adherent hES colonies were cultivated for 6 days in hES growth medium or medium supplemented with $0.1 \%$ dimethyl sulfoxide (DMSO) or $1 \mu \mathrm{M}$ RA. Cells were dislodged using dispase and transferred into suspension culture in hES medium. The following day, aggregates of differentiated cells were transferred to plates coated with $0.1 \%$ gelatin (Sigma-Aldrich, St. Louis, MO) and maintained in defined keratinocyte serum-free medium (dKSFM) (Invitrogen, Carlsbad, CA). For serial cultivation, subconfluent cells were harvested by trypsinization and re-plated onto gelatin-coated plates in dKSFM. Cell and colony morphology were documented using an Olympus IX-70 inverted microscope equipped with a DEI-750 camera (Optronics Engineering, Goleta, CA) and
Image-Pro Plus software (Media Cybernetics, Silver Spring, MD). Human epidermal keratinocytes were cultivated on murine feeder layers in standard keratinocyte culture medium as previously described $[18,19]$.

To generate three-dimensional tissues, cells were harvested by trypsinization and seeded at a density of $5 \times 10^{5} \mathrm{cells} / \mathrm{cm}^{2}$ onto a cellularized dermal matrix (Stratatech Corporation, Madison, WI) consisting of human dermal fibroblasts embedded in type I collagen in StrataLife media (Stratatech Corporation, Madison, WI) as previously described [19]. Cultures were kept submerged for 5 days, then raised to the air-interface and maintained for an additional 24 days to facilitate terminal differentiation. Tissue samples were prepared for histological evaluation as previously described.

\section{Reverse transcription-polymerase chain reaction (RT-PCR) analysis}

Total RNA was isolated using TRIzol Reagent according to the manufacturer's instructions (Invitrogen, Carlsbad, CA). Following DNase I treatment of $2 \mu \mathrm{g}$ total RNA using the DNA free kit (Ambion, Austin, TX), reverse transcription was performed using oligo dT primers and M-MLV reverse transcriptase (Invitrogen, Carlsbad, CA) per manufacturer's instructions. cDNA was analyzed by PCR amplification using the primer pairs listed in Supplementary Table 1. Amplified products were visualized by agarose gel electrophoresis and gene expression was confirmed by identification of the appropriate bands. GAPDH served as a loading control.

\section{Indirect immunofluorescence}

Cryopreserved sections were incubated for 1 hour at $37^{\circ} \mathrm{C}$ with primary antibody (Supplementary Table 2) diluted in blocking agent followed by incubation for 30 minutes at room temperature with goat anti-mouse or anti-rabbit IgG Alexa-594 secondary antibody (Invitrogen, Carlsbad, CA). Sections incubated with blocking agent served as controls for non-specific staining. Nuclei were counterstained with $5 \mu \mathrm{g} / \mathrm{ml}$ Hoechst 33258 in PBS. Digital image capture of individual fluorescence signals was performed using an Olympus IX-70 inverted fluorescent microscope equipped with Texas Red and Hoechst band pass filters, an Olympus DP70 digital camera, and DP Controller software (Olympus, Center Valley, PA). Identical image manipulations were performed for each set and dual-color images were created by overlay of single color captures of the same field.

\section{Barrier function analysis}

Electrical impedance, as measured by a Nova Derma Phase Meter (DPM) 9003 equipped with a DPM 9107 sensor probe (NOVA Technology Corporation, Gloucester, MA), was used to evaluate barrier function. The magnitude of impedance change during a defined measurement period correlates with the rate of transepidermal water loss [20], thus a large change in impedance as measured by DPM units is indicative of impaired barrier function. The probe was placed in direct contact with the tissue surface and impedance measurements were recorded at 0.5 second intervals for 10 seconds. hES-DK tissues resulting from three independent differentiation series were evaluated. For comparison studies, triplicate stratified squamous epithelial tissues generated from epidermal keratinocytes were analyzed. Three measurements were also taken from native skin. All values are reported as the mean \pm SD. Contiguous epithelialization of hES-DK tissues was confirmed by the presence of a uniformly-stained culture surface after exposure to 3-(4,5-dimethylthiazol-2-yl)-2,5-diphenyl tetrazolium 
Citation: Rasmussen CA, Schlosser SJ, Allen-Hoffmann BL (2014) Morphogenesis of Biologically Active Interfollicular Epidermis from Human Embryonic Stem Cell-derived Keratinocytes. J Stem Cell Res Ther 4: 163. doi:10.4172/2157-7633.1000163

Page 3 of 8

bromide, which is converted to a blue formazan product by metabolic activity.

\section{In vitro antimicrobial functional activity assay}

A total of three tissues from two independent differentiation experiments were evaluated for antimicrobial functionality. Biopsy punches $(8 \mathrm{~mm})$ of hES-DK tissue were placed epithelial side-down in individual wells of a 24 -well plate containing $200 \mu \mathrm{l}$ of serumfree keratinocyte medium [18], and incubated for 2 hrs at $37^{\circ} \mathrm{C}, 5 \%$ $\mathrm{CO}_{2}$ to extract protein. The antimicrobial assay was performed using Staphylococcus carnosus as previously described [21]. Media containing $0.4,0.8$, and $1.6 \mu \mathrm{l} / \mathrm{ml}$ of the host defense peptide LL37 were utilized as positive controls for antimicrobial activity, while the addition of medium only was used as a control for the extent of bacterial growth in the absence of treatment. The level of bacteria introduced into the assay, and the extent of bacterial growth with or without treatment, was determined by CFU determination. The CFU/ml was calculated according to formulation in the WASP2 spiral plater (Microbiology International, Frederick, MD) user manual. For each treatment, the extent of bacterial growth was normalized to the untreated control, which was defined as $100 \%$ bacterial growth. Data were analyzed by one-way ANOVA and pairwise comparisons between individual treatments and the bacterial growth control were completed using Dunnett's post-hoc test.

\section{Results}

\section{Retinoic acid-treated hES cells do not form stratified squamous epithelia in organotypic culture}

To determine if retinoic acid alone was sufficient to induce keratinocyte differentiation, the effects of RA treatment on gene expression and differentiation of hES cells were examined. After 6 days, both untreated (data not shown) and vehicle-treated control monolayer cultures (Figure 1A) contained fusiform, fibroblastic cells at the periphery of hES colonies. In contrast, a cobblestone, epithelial morphology was observed on the outer third of hES colonies treated with RA (Figure 1B). RA-treated hES cells were analyzed for gene expression of neural ectoderm and epithelial differentiation markers by RT-PCR (Figure 1C). Following RA treatment, expression of Oct4 mRNA decreased. RA has previously been reported to attenuate neural differentiation [22]. Although mRNA expression of the intermediate filament nestin remained unchanged in RA-treated cells, a reduction was observed for the neural ectoderm differentiation marker Sox 1 . mRNA expression of both $\Delta \mathrm{Np} 63$, a transcription factor expressed in the proliferative compartment of epithelial tissues, and keratin 18 (K18), an intermediate filament primarily associated with simple epithelia [23], increased with RA treatment. Expression of K14, an early marker of keratinocyte differentiation, was not induced by 6 days of RA treatment alone.

DMSO- and RA-treated hES cells were next introduced into organotypic culture to investigate the ability of these cells to form stratified tissue. Histological analysis revealed that DMSO-treated cells cultivated under organotypic conditions primarily formed wellorganized pseudostratified columnar epithelia (Figure 1D). In contrast, organotypic culture of RA-treated hES cells resulted in a disorganized collection of cells with no histological features characteristic of simple or stratified squamous epithelia (Figure 1E). Taken together these findings show that although RA induced expression of the epithelial markers $\triangle \mathrm{Np63}$ and K18 in hES cells, RA treatment alone did not promote K14 expression or induce differentiation of hES cells into an epithelial cell type capable of forming stratified squamous epithelia.

\section{hES-DK cells form keratinized stratified squamous epithelia displaying characteristics of the interfollicular epidermis}

Enrichment of RA-treated hES cultures for cells differentiating into the keratinocyte lineage was next explored. We used serial cultivation on gelatin-coated plates to select for cells exhibiting epithelial morphology. hES cells treated with DMSO or RA were transferred onto gelatin-coated culture plates and maintained in monolayer culture for two passages (Figure 2A). Typical of spontaneous differentiation, the cell population resulting from DMSO treatment was morphologically heterogeneous (data not shown). Conversely, after one passage onto gelatin-coated culture plates, RA-treated hES cells yielded a cell population, hES-derived epithelial (hES-DEp) cells, that exhibited a more flattened, epithelial morphology at the periphery of the expanding colony (Figure $2 \mathrm{~B}$ ). Harvest and subsequent passage of these hES-DEp cells onto gelatin-coated culture plates resulted in a population where the predominant cell type (hES-DK) exhibited a cobblestone morphology (Figure 2C) typical of epidermal keratinocytes (Figure 2D). These results strongly suggest that serial cultivation onto gelatin-coated plates served as a selection for cells with a keratinocyte phenotype.

hES-DEp cells, hES-DK cells, and human epidermal keratinocytes were then cultivated using organotypic conditions to assess the capacity of these cells to form stratified squamous epithelial tissue. The tissue architecture resulting from hES-DEp cells did not resemble stratified squamous epithelia and was disordered with numerous, atypical cells (Figure 2E). Surface coverage was incomplete and although limited regions displayed an epithelial appearance, highly vacuolized cells were observed throughout the tissue. In contrast, hES-DK cells formed a multi-layered stratified squamous epithelium (Figure 2F). Epithelial
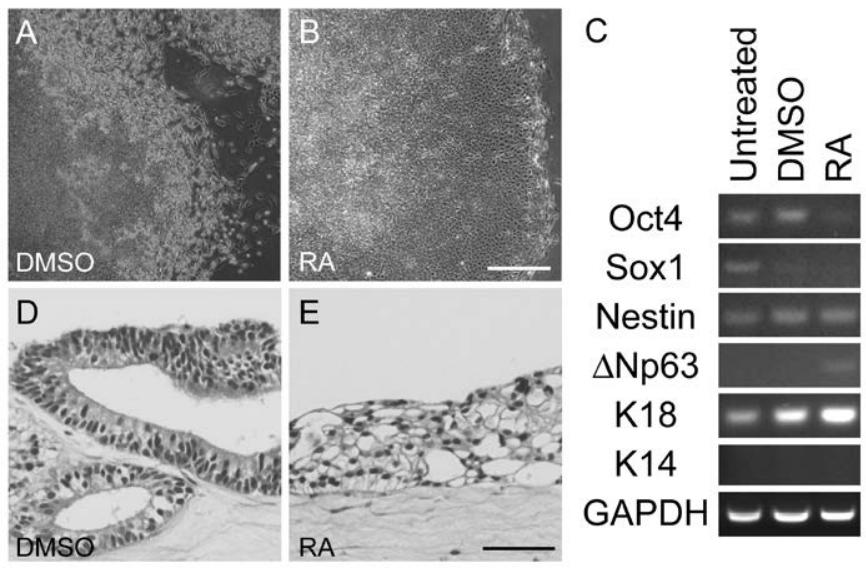

Figure 1: RA-treated hES cells express epithelial markers, but do not form organized structures when cultivated under organotypic conditions. Representative phase contrast images depicting the cell and colony morphology of hES cells after treatment with hES growth medium supplemented with DMSO (A) or RA (B). RT-PCR detection of neural or epithelial markers for untreated, DMSO-treated, and RA-treated samples (C). Results are representative of three independent experiments. Histological analysis of tissue generated from DMSO-treated hES cells revealed formation of well-organized pseudostratified columnar epithelia (D). Culture of RA-treated hES cells resulted in disorganized structures with limited areas of stratified epithelia (E). Scale bar represents $500 \mu \mathrm{m}$ (A and B); $50 \mu \mathrm{m}$ (D and $\mathrm{E})$. 
Citation: Rasmussen CA, Schlosser SJ, Allen-Hoffmann BL (2014) Morphogenesis of Biologically Active Interfollicular Epidermis from Human Embryonic Stem Cell-derived Keratinocytes. J Stem Cell Res Ther 4: 163. doi:10.4172/2157-7633.1000163

coverage was contiguous, although variation in the thickness of stratified hES-DK tissue was observed. Basal, spinous, and granular layers were evident throughout the tissue. Flattened, enucleated squames were present at the apical surface of stratified hES-DK tissue, however hESDK tissue lacked a well-defined stratum corneum. Squames were more frequently observed in thinner regions. Stratified squamous epithelia generated from human epidermal keratinocytes (Figure 2G) exhibited a consistent thickness with well-defined basal, spinous, granular and cornified layers.

To evaluate the progression of hES-DK cell development in monolayer culture, samples from each differentiation step were analyzed for gene expression of markers indicative of neural ectoderm and epithelial differentiation, as well as keratinocyte terminal differentiation (Figure 2H). Oct4 mRNA expression was not detected in hES-DEp and hES-DK monolayer cultures or in tissues generated by hES-DK cells. Sox 1 and nestin expression in hES-DEp cells was similar to RA-treated hES cells, but absent in hES-DK cells and hES-DK tissue. Compared to $\triangle \mathrm{Np} 63$ levels in stratified squamous epithelia generated from human epidermal keratinocytes, low levels of $\triangle \mathrm{Np} 63 \mathrm{mRNA}$ were detected at all stages of hES-DK differentiation. Robust K18 expression was observed in RA-treated, hES-DEp, and hES-DK cells, as well as in tissues generated by hES-DK cells or epidermal keratinocytes. K14 mRNA levels were low in the hES-DEp population; however K14 mRNA levels increased substantially in hES-DK cells. K14 levels in hESDK tissue were comparable to tissues generated with human epidermal keratinocytes. Expression of the late-stage keratinocyte differentiation markers involucrin and loricrin were first detected within hES-DK cells. These findings suggest that a defined cell population robustly expressing key markers of epidermal differentiation was required for epidermal morphogenesis.

\section{Proteins essential to the structure and function of keratinized stratified squamous epithelia are expressed and appropriately localized in hES-DK tissue}

hES-DK cell morphogenesis into stratified squamous epithelia prompted an investigation of the expression and localization of proteins necessary for the structure and function of stratified squamous epithelia. In these studies hES-DK tissues were compared to epidermal keratinocyte-generated tissues. E-cadherin appropriately localized to the membrane of cells positioned within the basal and immediately suprabasal layers (Figures $3 \mathrm{~A}$ and $3 \mathrm{~B}$ ), although staining was generally more intense in hES-DK tissue compared to stratified squamous epithelia generated from epidermal keratinocytes. P-cadherin protein expression was localized to the cell membrane and restricted to cells of the basal and immediately suprabasal layers (Figures 3C and 3D), although staining appeared substantially diminished in hESDK tissues relative to epidermal keratinocyte-generated tissue. The $\beta$-catenin expression pattern (Figures $3 \mathrm{E}$ and $3 \mathrm{~F}$ ) was similar to that of E-cadherin and no nuclear localization was detected. The expression of keratin intermediate filaments is highly regulated and dependent upon the stage of keratinocyte terminal differentiation [24]. Typical for stratified squamous epithelia, the expression of K14 protein was found in basal and suprabasal layers of tissues generated from hESDK and epidermal keratinocytes (Figures $3 \mathrm{G}$ and $3 \mathrm{H}$ ). For all tissues examined, $\mathrm{K} 1$ was appropriately localized to the suprabasal layers (Figures 3I and 3J) while keratin 2 (K2) was properly restricted to the upper spinous and granular layers (Figures $3 \mathrm{~K}$ and $3 \mathrm{~L}$ ). Nuclear localization of the transcription factor $\mathrm{p} 63$ was detected exclusively in cells of the basal layer in hES-DK and control epidermal keratinocyte tissues (Figures $3 \mathrm{M}$ and $3 \mathrm{~N}$ ). Punctuate staining for filaggrin, a latestage keratinocyte terminal differentiation marker, was found in the cytosol of cells located in the granular layer for hES-DK and human epidermal keratinocyte-derived tissues (Figures $3 \mathrm{O}$ and 3P). Similarly, keratinocyte type 1 transglutaminase (Tgase1) was properly localized to the cell membrane of the spinous and granular layers (Figures $3 \mathrm{Q}$ and 3R). These results demonstrated that key structural proteins of stratified squamous epithelia, and proteins associated with both earlyand late-stage keratinocyte terminal differentiation, were appropriately expressed and localized in hES-DK tissues relative to stratified squamous epithelia generated from epidermal keratinocytes.

\section{hES-DK stratified squamous epithelia do not exhibit cutaneous barrier function}

Human skin maintains tissue homeostasis by providing a barrier to transepidermal water loss. To evaluate the level of cutaneous barrier function of hES-DK and human epidermal keratinocyte tissues, skin surface electrical impedance was measured. Stratified squamous epithelial tissue generated from epidermal keratinocytes yielded impedance values similar to that of native skin indicating the presence of cutaneous barrier function (Figure 4). In contrast, hESDK tissue did not form an epidermal permeability barrier. To ensure all tissues possessed contiguous epithelialization, which is critical for this assay, hES-DK tissues were incubated with the metabolic dye 3-(4,5-dimethylthiazol-2-yl)-2,5-diphenyl tetrazolium bromide [25] which confirmed complete culture surface coverage (data not shown). Since the barrier to transepidermal water loss results primarily from

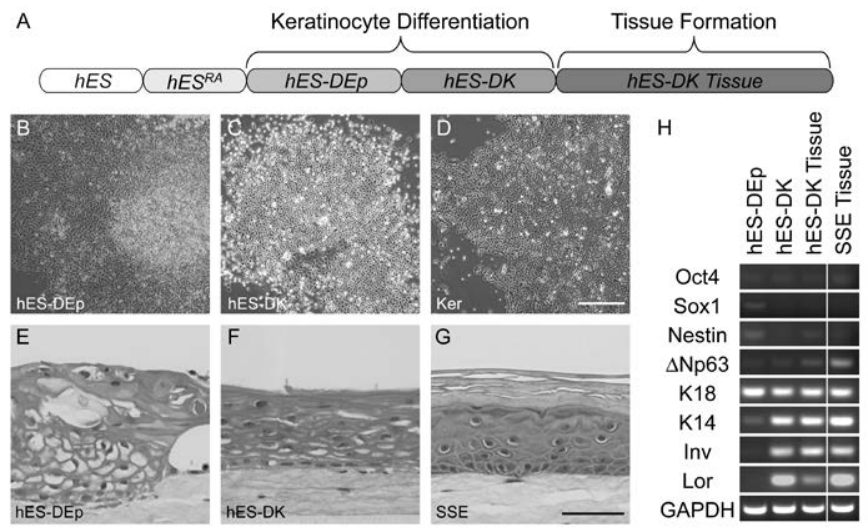

Figure 2: hES-DK cells terminally differentiate and form keratinized stratified squamous epithelia. Schematic identifying each step of hESDK differentiation (A). hES cells treated with RA (hESRA) were harvested, maintained in suspension overnight, and passaged onto gelatin-coated plates in dKSFM. These cells (hES-DEp) were harvested prior to confluence and passaged once more onto gelatin-coated plates in dKSFM (hES-DK). Subconfluent hES-DK cells were harvested and seeded into organotypic culture for tissue formation. Representative phase contrast images of subconfluent hES-DEp (B), hES-DK (C), and epidermal keratinocytes (D). Histological analysis revealed the tissue architecture generated from organotypic culture of hES-DEp cells $(E)$, hES-DK cells $(F)$, and epidermal keratinocytes $(G)$. RT-PCR detection of neural, epithelial, and keratinocyte terminal differentiation markers during hES-DK differentiation $(\mathrm{H})$. Samples from hES-DEp and hES-DK cells, and the tissue generated from hES-DK cells, were evaluated. Results depict one of three independent differentiation series. Expression of Sox1 and nestin were confirmed by evaluating spontaneously differentiated hES cells. Stratified squamous epithelia (SSE) generated from epidermal keratinocytes were included as a comparison for known keratinocyte markers. Scale bar represents $500 \mu \mathrm{m}$ (B,C,D); $50 \mu \mathrm{m}$ $(\mathrm{E}, \mathrm{F}, \mathrm{G})$. epidermal keratinocytes (Ker), keratin 18 (K18), keratin 14 (K14), involucrin (Inv), loricrin (Lor). 


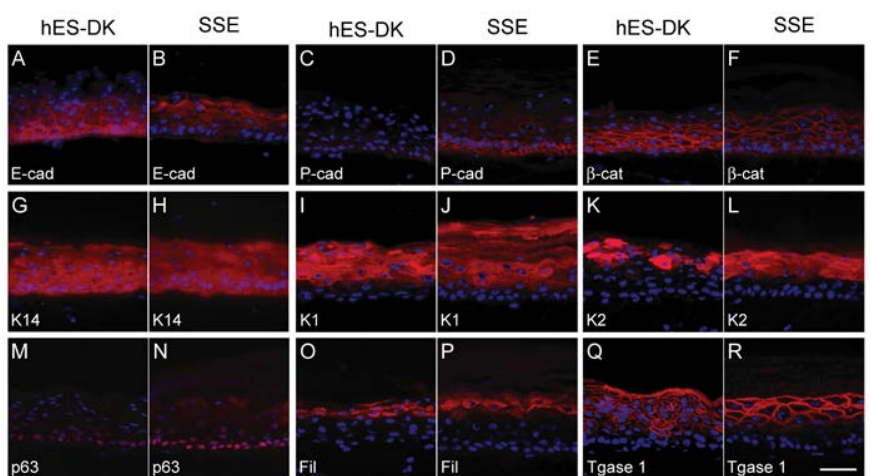

Figure 3: hES-DK tissue exhibits expression and appropriate localization of proteins critical to the structure and function of keratinized stratified squamous epithelia. Indirect immunofluorescent detection (red) of E-cadherin (A and $B$ ), P-cadherin (C and $D)$, $\beta$-catenin ( $E$ and $F) ~ K 14$ ( $G$ and $H), K 1$ ( $\mathrm{I}$ and $\mathrm{J}), \mathrm{K} 2$ ( $\mathrm{K}$ and $\mathrm{L}$ ), p63 ( $\mathrm{M}$ and $\mathrm{N}$ ), filaggrin ( $\mathrm{O}$ and $\mathrm{P}$ ), and Tgase1 ( $\mathrm{Q}$ and $\mathrm{R})$ in stratified tissue generated from hES-DK and epidermal keratinocytes. Hoechst 33258 stain was used to visualize nuclei (blue). Three independent experiments yielded comparable expression patterns. Scale bar represents $50 \mu \mathrm{m}$. E-cadherin (E-Cad), P-cadherin (P-Cad), $\beta$-catenin ( $\beta$-cat), filaggrin (fil).

lipid deposition in the intercellular spaces between the enucleated squames of the stratum corneum, these findings were consistent with the histological observation noting the lack of a well-defined cornified layer in hES-DK tissue.

Stratified squamous epithelia generated from hES-DK cells express proteins associated with innate immunity and demonstrate antimicrobial activity in vitro

HDPs are integral to the innate immunity of epithelial tissues (reviewed in [26]), including oral and epidermal stratified squamous epithelia, and function as a barrier against entry of infectious agents in stratified human tissues [27-29]. In human skin, both human $\beta$ defensin 3 (hBD3) and human cathelicidin (LL37) are typically stored in lamellar bodies within differentiated keratinocytes [30,31]. To determine if HDPs were expressed in hES-DK stratified squamous epithelia, the expression patterns of hBD3 and LL37 were examined. hBD3 protein was localized to the granular layer in hES-DK tissue (Figure 5A), consistent with the pattern observed in stratified squamous epithelia generated from epidermal keratinocytes (Figure 5B). Staining for LL37 protein was observed in all layers of hES-DK tissue (Figure 5C). Similarly, LL37 was distributed throughout tissues generated from human epidermal keratinocytes, however slightly more intense signal was noted in the basal and immediately suprabasal layers (Figure 5D).

The presence of HDPs in hES-DK tissue implied the potential for innate immune system functionality, therefore an in vitro assay was used to evaluate antimicrobial activity. In this assay bacterial levels were shown to significantly decrease in a dose-dependent manner upon treatment with increasing concentrations of purified LL37 peptide (Figure 5E). Samples treated with $0.4 \mu \mathrm{g} / \mathrm{ml} \mathrm{LL37} \mathrm{peptide}$ resulted in a $43 \%$ inhibition in bacterial growth when compared to the level of bacterial growth found in untreated control samples, indicative of bacteriostatic activity. Similarly, media conditioned by hES-DK stratified squamous epithelia significantly inhibited bacterial growth by $39 \%$ compared to the bacterial growth control. Detection of antimicrobial activity confirmed formation of a biologically functional stratified squamous epithelia and represents the first description of this activity in a hES cell-derived three-dimensional tissue.

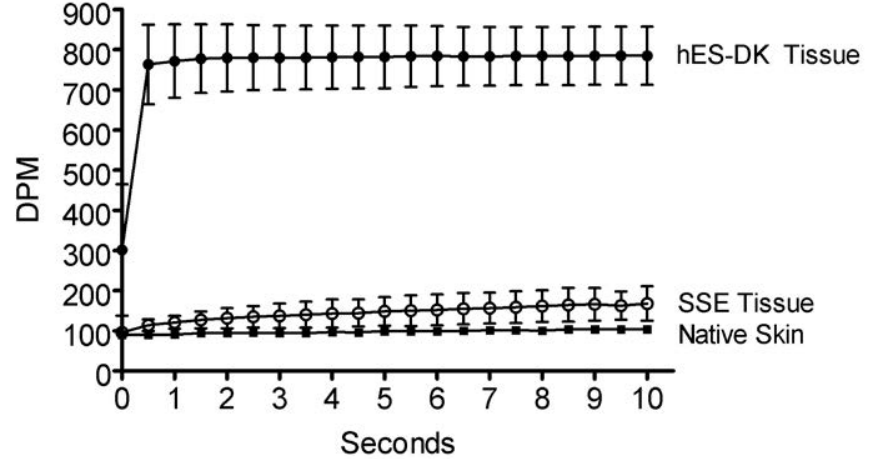

Figure 4: hES-DK tissue does not exhibit cutaneous barrier function. Barrier function properties of stratified tissues as measured by skin surface electrical impedance (DPM units). Unlike stratified squamous epithelia (SSE) generated from epidermal keratinocytes, hES-DK tissue did not possess cutaneous barrier function similar to native skin. Data represent mean \pm standard deviation.
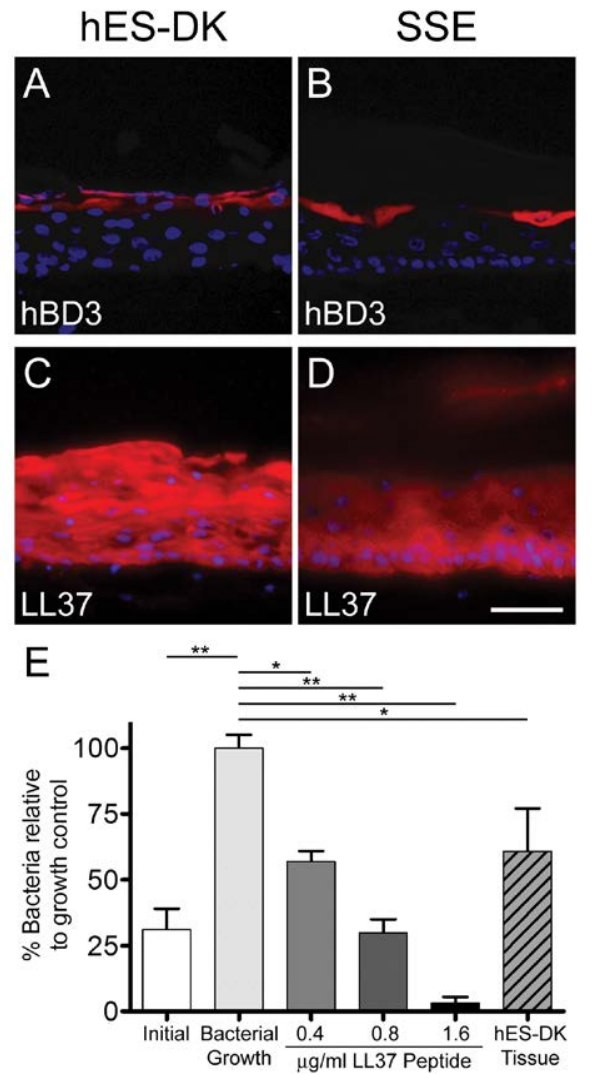

Figure 5: Functional host defense peptides are expressed and appropriately localized in hES-DK tissue, as confirmed by antimicrobial activity in vitro against $S$. carnosus. Indirect immunofluorescent detection (red) of the host defense peptides hBD3 (A and B) and LL-37 (C and D) in stratified tissue generated from hES-DK and epidermal keratinocytes. Hoechst 33258 stain was used to visualize nuclei (blue). Results are representative of three independent experiments. Scale bar represents $50 \mu \mathrm{m}$. Extent of bacterial growth in the absence or presence of antimicrobial peptide or hES-DK tissue conditioned medium (E). Compared to the initial bacterial concentration, significant growth was observed in the bacterial growth control samples. The addition of LL37 peptide and conditioned medium significantly reduced the level of bacterial growth as compared to untreated control samples $\left({ }^{*} p<0.05\right.$; $\left.{ }^{* *} p<0.01\right)$. Data represent mean \pm standard deviation. 


\section{Discussion}

To successfully restore damaged tissues, allogeneic stem cell therapies for the treatment of severe cutaneous injuries require the derived cell types to generate biologically functional keratinized stratified squamous epithelial tissue. hES- and hiPS-derived cells have been reported to generate stratified epithelia in vitro with varying levels of success [7,9,14-16]. To date, no functional bioactivities for stratified squamous epithelia generated using pluripotent cellderived sources have been reported. Here we evaluated the capacity of hES-derived epithelial cell populations to form organized structure, thoroughly characterized the expression patterns of proteins essential for keratinized stratified squamous epithelia structure and function, and assessed key bioactivities typical of the interfollicular epidermis.

As first described by Metallo et al. [6], treatment of hES cells with RA to initiate epithelial differentiation and BMP to inhibit neural differentiation enhanced differentiation efficiency of cells displaying an epithelial-like morphology. Our studies confirm and expand upon the role of RA in the generation of hES-DK cells. We found that mRNA expression of epithelial differentiation markers $(\Delta \mathrm{Np} 63, \mathrm{~K} 18)$ in RA-treated cells was upregulated in comparison to DMSO-treated or untreated hES cell controls, while neural ectoderm differentiation marker expression (nestin, Sox 1) remained unaffected or was attenuated. Expression of K14 mRNA was not detected in RA-treated cells. Our findings replicate those recently reported by Leydon et al. [16] where p63+, K18+, K14- simple epithelial cells were generated from hES cells using nearly identical methods. Although RA supported differentiation of a simple epithelial cell type, it appears insufficient to induce keratinocyte differentiation. We further found that RAtreated hES cells cultured under organotypic conditions formed tissue that lacked organized structure. This is in contrast to Leydon's results where p63+, K18+, K14- RA-treated hES cells developed a uniform multilayered, non-keratinized squamous epithelium when cultivated on a vocal cord fibroblast-containing dermal matrix. Leydon and coworkers also demonstrated that these same cells failed to form threedimensional structure when a non-cellularized collagen matrix was used, implying that stromal-derived paracrine signaling was required for three-dimensional tissue formation. Our studies, which utilized a human dermal fibroblast-populated dermal matrix, suggest that the signaling factors supplied by dermal fibroblasts were inadequate to promote the formation of organized tissue from RA-treated hES cells.

RA-treated hES cell aggregates attached to the culture surface and supported the outgrowth of hES-DEp cells exhibiting a cuboidal, epithelial morphology. Selection by subsequent passage of hES-DEp cells onto gelatin-coated culture plates resulted in clonal growth of hES-DK cells which exhibited epidermal keratinocyte morphology and, unlike hES-DEp cells, formed keratinized stratified squamous epithelia in organotypic culture. Gene expression analysis was completed at each step of differentiation and hES-DK tissue formation to assess changes in epidermal marker expression. A low level of $\triangle$ Np63 mRNA was detected at all stages of differentiation, consistent with its function in the maintenance of epithelial proliferation [32]. Similarly K18, commonly associated with simple epithelia, was expressed throughout hES-DK differentiation. Expression of K14, first evident in the hES-DEp population, became more pronounced after serial cultivation and subsequent organotypic culturing. Although previous studies required prolonged exposure to differentiation agents [8], clonal isolation and expansion [9], or extended serial cultivation $[6,15]$ to enrich for a K14+ keratinocyte-like population, we found that a single serial cultivation step onto gelatin-coated plates was sufficient to substantially enrich for cells expressing markers of a keratinocyte lineage. Integrin-mediated adhesion signaling plays critical roles in stem-cell-niche interactions [33], as well as keratinocyte proliferation and differentiation (reviewed in [34]). Since adhesion to gelatin during cultivation enhanced selection for cells with keratinocyte properties, it is possible that integrin-mediated signaling may also be critical for keratinocyte lineage differentiation. In addition to K14, expression of involucrin and loricrin, components of the keratinocyte cornified envelope, were detected in both hES-DK cells and stratified tissue. It is interesting to note that the succession of gene expression acquired as hES-derived populations adopted a keratinocyte phenotype paralleled the progression of epidermal marker expression found during fetal epidermal morphogenesis [35,36]. Importantly, we found that expression of key keratinocyte markers, most notably K14, was predictive of the cells' ability to form a keratinized stratified squamous epithelium.

Within the interfollicular epidermis, proteins associated with cellcell adhesion and keratinocyte terminal differentiation are expressed and localized to specific layers. Although examination of several of these proteins in hES cell-derived tissue constructs has previously been described $[9,14,15]$, a comprehensive examination of proteins essential for the structure and function of keratinized stratified squamous epithelia has not yet been reported. Our study provides the first examination of the cell-cell adhesion proteins E-cadherin, P-cadherin, and $\beta$-catenin in three-dimensional hES-derived epithelia. E-cadherin and $\beta$-catenin staining was cell membrane-associated, localized to the basal and immediately suprabasal layers, and more pronounced in hES-DK tissue relative to epidermal keratinocyte-derived tissue. Upregulation of these proteins could inhibit keratinocyte terminal differentiation [37], contributing to the morphological differences noted between hES-DK and human epidermal keratinocyte-generated tissues. Although substantially reduced relative to tissues generated using epidermal keratinocytes, P-cadherin staining in hES-DK tissue was cell membrane-associated and localized to the basal and immediately suprabasal layers. This pattern is characteristic of the epidermis, but not of non-keratinized stratified squamous mucosal epithelia where P-cadherin expression is found throughout the suprabasal layers [38,39]. Keratin expression patterns not only change as terminal differentiation proceeds, but reflect tissue specificity. Although K14 is found in many epithelial tissues, $\mathrm{K} 1$ is differentiationspecific and primarily associated with keratinized stratified squamous epithelia [40]. Both K14 and K1 expression was observed suggesting morphogenesis of a keratinized tissue. In Dabelsteen et al. [14] and Metallo et al. [15], expression of keratin 10 (K10), the partner of K1, was absent or displayed a localization pattern that differed considerably from that of epidermal keratinocyte-generated tissues. In our study, K1 expression was appropriately restricted to the immediately suprabasal layers, similar to the K10 expression pattern observed by Guenou and coworkers [9]. We found that the epidermal-specific keratin K2, which has not previously been examined in hES-derived stratified tissues. $\mathrm{K} 2$, typically restricted to the upper spinous and granular layers of the epidermis and not expressed in other keratinized stratified squamous epithelial tissues, is expressed to varying levels in different regions of the body during fetal skin development [41]. The level and variability of $\mathrm{K} 2$ protein expression observed in hES-DK tissue are consistent with this pattern. p63 expression in hES-DK tissue was restricted to the basal layer, unlike the findings of Dabelstein et al. or Metallo et al. $[14,15]$ where $\mathrm{p} 63$ was dispersed throughout the tissue as is commonly 
found in neoplastic epithelia [42,43]. Expression of transglutaminase 1 , which functions to crosslink proteins into the cornified envelope during keratinocyte terminal differentiation, was also found to be appropriately localized. Filaggrin, which is integral to the epidermal permeability barrier [44], was properly restricted to the granular layer in hES-DK tissue. This supports the earlier finding of Guenou and coworkers [9] but stands in contrast to Metallo et al. [15] where filaggrin in hES-derived epithelial tissues was virtually absent. The presence and appropriate localization of proteins essential for the structure and function of keratinized stratified squamous epithelia, and specifically the epidermis, confirms morphogenesis of interfollicular epidermal tissue from hES-derived keratinocytes.

In vivo the epidermis serves as a barrier to transepidermal water loss, maintaining fluid homeostasis via cutaneous barrier function, and as a barrier to infectious agents, producing HDPs integral to innate immunity. Evaluation of these biological activities in epithelial tissue generated from a pluripotent stem cell source has not previously been reported. Cutaneous barrier function is generated by deposition of lipids in the intercellular spaces between cells of the cornified envelope in the stratum corneum [45-47]. Unlike native human skin or tissue formed from human epidermal keratinocytes, we found that hES-DK tissues did not possess a functional permeability barrier most likely resulting from the lack of a well-defined stratum corneum. It is possible that extending the period of air-interface cultivation would promote further accumulation of enucleated squames, establish a more substantial stratum corneum, and enhance the development of cutaneous barrier function. hBD3 protein, a HDP associated with the more terminallydifferentiated layers of the epidermis [28], was found to localize to the granular layer in both hES-DK and human epidermal keratinocytegenerated tissue. Protein expression of the cathelicidin LL37 was primarily restricted to the basal and immediately supra-basal layers in tissues generated from human epidermal keratinocytes. In contrast, intense staining for LL37 was detected throughout hES-DK tissue. The reason for this difference is not currently known. The presence of HDPs implied the potential for innate immune system functionality in hES-DK stratified squamous epithelia, therefore antimicrobial activity was evaluated. Bacteriostatic activity was confirmed for hES-DK tissue, which inhibited bacterial growth by $39 \%$. This level of antimicrobial activity is comparable to that found previously for tissue generated from an epidermal keratinocyte source using the same organotypic cultivation process [21]. Further investigation into the biological activities of hES-derived tissue is required to understand the correlation between antimicrobial activity and specific HDPs. The bioactivity displayed by hES-DK tissue represents the first demonstration of biological activity in a hES-derived stratified squamous epithelial tissue and the first description of antimicrobial activity in a three-dimensional tissue generated from a hES cell-derived source.

Ultimately the clinical utility of pluripotent stem cells relies on the ability to direct differentiation into the desired cell lineage, facilitate appropriate tissue formation, and confirm tissue-specific biological activity. Such allogeneic cell-based therapies offer a means to restore viability and function to severely damaged cutaneous tissues. In this study, we report the morphogenesis of biologically active interfollicular epidermis from hES-derived keratinocytes. The progression of gene expression found during keratinocyte lineage differentiation closely mimicked the orderly sequence of epidermal marker expression found during fetal epidermal morphogenesis. Specific protein localization confirmed appropriate tissue organization and hES cell-derived interfollicular epidermal tissue was shown to possess antimicrobial activity, a key characteristic of epidermal function. The successful generation of biologically active hES-derived stratified squamous epithelia demonstrates a significant advance in the development of a bioengineered human organ for clinical use.

\section{Acknowledgements}

We thank the guidance and technical support of Dr. Ted Golos and his staff, in particular Behzad Gerami-Naini and Maria Giakoumopoulos, during the course of these studies. We also wish to thank Christina Thomas-Virnig for editorial assistance with this manuscript and Satoshi Kinoshita for the processing of histological samples.

\section{References}

1. Murry CE, Keller G (2008) Differentiation of embryonic stem cells to clinically relevant populations: lessons from embryonic development. Cell 132: 661-680.

2. Morris SA, Daley GQ (2013) A blueprint for engineering cell fate: current technologies to reprogram cell identity. Cell Res 23: 33-48.

3. Green H, Easley K, luchi S (2003) Marker succession during the development of keratinocytes from cultured human embryonic stem cells. Proc Natl Acad Sci U S A 100: 15625-15630.

4. Ji L, Allen-Hoffmann BL, de Pablo JJ, Palecek SP (2006) Generation and differentiation of human embryonic stem cell-derived keratinocyte precursors. Tissue Eng 12: 665-679.

5. Iuchi S, Dabelsteen S, Easley K, Rheinwald JG, Green H (2006) Immortalized keratinocyte lines derived from human embryonic stem cells. Proc Natl Acad Sci U S A 103: 1792-1797.

6. Metallo CM, Ji L, de Pablo JJ, Palecek SP (2008) Retinoic acid and bone morphogenetic protein signaling synergize to efficiently direct epithelial differentiation of human embryonic stem cells. Stem Cells 26: 372-380.

7. Itoh M, Kiuru M, Cairo MS, Christiano AM (2011) Generation of keratinocytes from normal and recessive dystrophic epidermolysis bullosa-induced pluripotent stem cells. Proc Natl Acad Sci U S A 108: 8797-8802.

8. Kidwai FK, Liu H, Toh WS, Fu X, Jokhun DS, et al. (2013) Differentiation of human embryonic stem cells into clinically amenable keratinocytes in an autogenic environment. J Invest Dermatol 133: 618-628.

9. Guenou H, Nissan X, Larcher F, Feteira J, Lemaitre G, et al. (2009) Human embryonic stem-cell derivatives for full reconstruction of the pluristratified epidermis: a preclinical study. Lancet 374: 1745-1753.

10. Lemaître G, Nissan X, Baldeschi C, Peschanski M (2011) Concise review: Epidermal grafting: the case for pluripotent stem cells. Stem Cells 29: 895-899.

11. MacNeil S (2007) Progress and opportunities for tissue-engineered skin. Nature 445: 874-880.

12. Moharamzadeh K, Brook IM, Van Noort R, Scutt AM, Thornhill M H (2007) Tissue-engineered oral mucosa: a review of the scientific literature. J Dent Res 86: $115-124$

13. Mansbridge JN (2009) Tissue-engineered skin substitutes in regenerative medicine. Curr Opin Biotechnol 20: 563-567.

14. Dabelsteen S, Hercule P, Barron P, Rice M, Dorsainville G, et al. (2009) Epithelial cells derived from human embryonic stem cells display p16INK4A senescence, hypermotility, and differentiation properties shared by many P63+ somatic cell types. Stem Cells 27: 1388-1399.

15. Metallo CM, Azarin SM, Ji L, de Pablo JJ, Palecek SP (2009) Human embryonic stem cell-derived keratinocytes exhibit an epidermal transcription program and undergo epithelial morphogenesis in engineered tissue constructs. Tissue Eng Part A 16: 213-223

16. Leydon C, Selekman JA, Palecek S, Thibeault SL (2013) Human embryonic stem cell-derived epithelial cells in a novel in vitro model of vocal mucosa Tissue Eng Part A 19: 2233-2241.

17. Thomson JA, Itskovitz-Eldor J, Shapiro SS, Waknitz MA Swiergiel JJ, et al. (1998) Embryonic stem cell lines derived from human blastocysts. Science 282: $1145-1147$

18. Allen-Hoffmann BL, Schlosser SJ, Ivarie CA, Sattler CA, Meisner LF, et al (2000) Normal growth and differentiation in a spontaneously immortalized neardiploid human keratinocyte cell line, NIKS. J Invest Dermatol 114: 444-455.

19. Rasmussen CA, Gibson AL, Schlosser SJ, Schurr MJ, Allen-Hoffmann 
Citation: Rasmussen CA, Schlosser SJ, Allen-Hoffmann BL (2014) Morphogenesis of Biologically Active Interfollicular Epidermis from Human Embryonic Stem Cell-derived Keratinocytes. J Stem Cell Res Ther 4: 163. doi:10.4172/2157-7633.1000163

Page 8 of 8

BL (2010) Chimeric composite skin substitutes for delivery of autologous keratinocytes to promote tissue regeneration. Ann Surg 251: 368-376.

20. Gabard B, Treffel P, Corneum P Elsner, Berardesca E, Maibach HI (1994) Hardware and Measuring Principle: The Nova DPM 9003, in Bioengineering of the Skin: Water and the Stratum Editor. CRC Press: Boca Raton, USA, 177195

21. Thomas-Virnig CL, Centanni JM, Johnston CE, He LK, Schlosser SJ, et al. (2009) Inhibition of multidrug-resistant Acinetobacter baumannii by nonviral expression of hCAP-18 in a bioengineered human skin tissue. Mol Ther 17 562-569.

22. Pankratz MT, Li XJ, Lavaute TM, Lyons EA, Chen X, et al. (2007) Directed neural differentiation of human embryonic stem cells via an obligated primitive anterior stage. Stem Cells 25: 1511-1520.

23. Owens DW, Lane EB (2003) The quest for the function of simple epithelial keratins. Bioessays 25: 748-758.

24. Fuchs E (1995) Keratins and the skin. Annu Rev Cell Dev Biol 11: 123-153.

25. Kidd DA, Johnson M, Clements J (2007) Development of an in vitro corrosion/ irritation prediction assay using the EpiDerm skin model. Toxicol In Vitro 21 1292-1297.

26. Wiesner J, Vilcinskas A (2010) Antimicrobial peptides: the ancient arm of the human immune system. Virulence 1: 440-464.

27. Nishimura M, Abiko Y, Kusano K, Yamazaki M, Saitoh M, et al. (2003) Localization of human beta-defensin 3 mRNA in normal oral epithelium, leukoplakia, and lichen planus: an in situ hybridization study. Med Electron Microsc 36: 94-97.

28. Supp DM, Karpinski AC, Boyce ST (2004) Expression of human beta-defensins HBD-1, HBD-2, and HBD-3 in cultured keratinocytes and skin substitutes. Burns 30: 643-648.

29. Frohm Nilsson M, Sandstedt B, Sorensen O, Weber G, Borregaard N, et al. (1999) The human cationic antimicrobial protein (hCAP18), a peptide antibiotic is widely expressed in human squamous epithelia and colocalizes with interleukin-6. Infect Immun 67: 2561-2566.

30. Braff MH, Di Nardo A, Gallo RL (2005) Keratinocytes store the antimicrobia peptide cathelicidin in lamellar bodies. J Invest Dermatol 124: 394-400.

31. Steinstraesser L, Koehler T, Jacobsen F, Daigeler A, Goertz O, et al. (2008) Host defense peptides in wound healing. Mol Med 14: 528-537.

32. Carroll DK, Brugge JS, Attardi LD (2007) p63, cell adhesion and survival. Cell Cycle 6: 255-261.
33. Ellis SJ, Tanentzapf G (2010) Integrin-mediated adhesion and stem-cell-niche interactions. Cell Tissue Res 339: 121-130.

34. Margadant C, Charafeddine RA, Sonnenberg A (2010) Unique and redundant functions of integrins in the epidermis. FASEB J 24: 4133-4152.

35. Akiyama M, Smith LT, Yoneda K, Holbrook KA, Hohl D, et al. (1999) Periderm cells form cornified cell envelope in their regression process during human epidermal development. J Invest Dermatol 112: 903-909.

36. Müller EJ, Williamson L, Kolly C, Suter MM (2008) Outside-in signaling through integrins and cadherins: a central mechanism to control epidermal growth and differentiation? J Invest Dermatol 128: 501-516.

37. Muñoz-Guerra MF, Marazuela EG, Fernández-Contreras ME, Gamallo C (2005) P-cadherin expression reduced in squamous cell carcinoma of the oral cavity: an indicatior of poor prognosis. Cancer 103: 960-969.

38. The Human Protein Atlas.

39. Chu PG, Weiss LM (2002) Keratin expression in human tissues and neoplasms. Histopathology 40: 403-439.

40. Smith LT, Underwood RA, McLean WH (1999) Ontogeny and regiona variability of keratin $2 \mathrm{e}(\mathrm{K} 2 \mathrm{e})$ in developing human fetal skin: a unique spatia and temporal pattern of keratin expression in development. $\mathrm{Br} \mathrm{J}$ Dermatol 140 582-591.

41. Bircan S, Candir O, Kapucoglu N, Baspinar S (2006) The expression of p63 in basal cell carcinomas and association with histological differentiation. J Cutan Pathol 33: 293-298.

42. Westfall MD, Pietenpol JA (2004) p63: Molecular complexity in development and cancer. Carcinogenesis 25: 857-864.

43. McGrath JA, Uitto J (2008) The filaggrin story: novel insights into skin-barrier function and disease. Trends Mol Med 14: 20-27.

44. Nemes Z, Steinert PM (1999) Bricks and mortar of the epidermal barrier. Exp Mol Med 31: 5-19.

45. Ponec M, Gibbs S, Pilgram G, Boelsma E, Koerten H, et al. (2001) Barrie function in reconstructed epidermis and its resemblance to native human skin. Skin Pharmacol Appl Skin Physiol 14: 63-71.

46. Ponec M, Weerheim A, Kempenaar J, Mulder A, Gooris GS, et al. (1997) The formation of competent barrier lipids in reconstructed human epidermis requires the presence of vitamin C. J Invest Dermatol 109: 348-355.

47. Dale BA, Holbrook KA, Kimball JR, Hoff M, Sun TT (1985) Expression of epidermal keratins and filaggrin during human fetal skin development. $\mathrm{J}$ Cell Biol 101: 1257-1269. 